\title{
Effect of electron-magnon coupling on the electronic spectrum of weakly doped high- $T_{C}$ compounds
}

\author{
M. A. Ivanov \\ Kurdyumov Institute of Metal Physics of the Ukrainian National Academy of Sciences, \\ 36 Vernadskii Prs., 252142 Kiev-142, Ukraine \\ V. M. Loktev \\ Bogolyubov Institute for Theoretical Physics of the Ukrainian National Academy of Sciences, \\ 14-b Metrologichna Str., 252143 Kiev-143, Ukraine \\ E-mail: vloktev@gluk.apc.org \\ Yu. G. Pogorelov \\ Departamento de Fisica, Universidad do Porto, 4150 Porto, Portugal \\ Received March 2, 1998
}

\begin{abstract}
A theoretic analysis of magnon-induced damping $\Gamma$ of quasiparticle states in the normal phase of doped copper-oxide high- $T_{c}$ materials is developed, based on a microscopic model which accounts for the specific $2 D$ structure of their electron and magnon spectra. Among the obtained energy and temperature dependences of $\Gamma$ in different regimes, the most peculiar is the anomalously early onset of linear temperature dependence $\Gamma \propto T$ with a doping-independent coefficient.
\end{abstract}

PACS: 72.10.Di, 74.20.Mn, 74.25.Fy

\section{Introduction}

The studies of coexistence and interplay between magnetic and electric properties of layered copper oxides are one of the most immediate problems in high- $T_{c}$ superconductivity (HTSC). The general scenario for development of phase states in these systems with their doping was already established from the very early experimental data [1]. The initial compounds are AFM-ordered Mott insulators with sufficiently high Neel temperature $T_{N}$, but this long-range magnetic order is very rapidly lost under small doping $c$, as evidenced by a sharp drop of $T_{N}$ with $c$ (until the AFM phase gets absorbed by the low temperature spin-glass and futher spin-liquid phases); this process is followed by the insulator-metal transition. However, the metallic phase of HTSC compounds cannot be considered as ordinary paramagnetic one in the usual sense, since it still preserves the short-range AFM order which is clearly revealed in its either static and dynamic properties (see, e.g., Refs. 2-6 and also the reviews $[7,8])$. It should be stressed that, in copper-oxide materials, unlike common magnetic metals, there are two different kinds of fermions responsible for the conducting and magnetic properties. The conductivity is mainly determined by the charge carriers (holes, either mobile or localized) from the doping, whereas the strong magnetic correlations are mainly produced by the localized «core» spins. Also, these two kinds of particles are spatially separated: the carriers predominantly occupy the oxygen sites, and the core spins are those at copper sites. Notably, the destroying of long-range magnetic order in cuprate $\mathrm{CuO}_{2}$ layers preceedes the appearance of metallic conductivity in them; hence, at low concentration $c$ of dopants, the HTSC compounds are still typical doped semiconductors with shallow acceptor levels and hopping type of conductivity (in particular, for the lanthanum system this is true at $c<5 \%$, while the acceptor binding energy is $\left.\varepsilon_{0} \approx 35 \mathrm{meV}[9,10]\right)$. It follows from the aforesaid that the proper theoretic description of the breakdown of magnetic order and of the insulator-metal transition in HTSC (at least, for small $c$ ) perhaps cannot be based on commonly used translationallyinvariant models. (The very number of such models, including different magnetic mechanisms of pairing, 
is too long to be counted, many of them are discussed in the reviews, Refs. 11-15.)

At the same time, attempts have been made [16,17] (see also Refs. 18,19) to consider these problems, starting from the primordial importance of disordered (impurity) character of weakly doped HTSC compounds. Initially, mainly due to the change of the oxygen component, the acceptor levels with localized holes on them appear in AFMordered cuprate planes (this localization is greatly facilitated by the striking $2 D$ character of HTSC electronic properties). At low $c$, these «impurity» holes give rise to a disordered spin subsystem on which the regular spin excitations (magnons) are scattered and hence damped. As a result, the longrange magnetic order gets destroyed and there appears such a minimum value $k_{\min }$ of wavevector that the magnons with $k<k_{\min }$ cannot exist because of strong damping. At lower concentrations this value is estimated as $k_{\min } \sim c a^{-1}$, but at higher concentrations (metallic ones including (see Ref. 20)) it is changed for $k_{\min } \sim \sqrt{c / c_{f}} \lambda_{B}^{-1}$, where $\lambda_{B}$ is the Bloch domain wall width and $c_{f}$ is the concentration where the decaying $T_{N}(c)$ meets the freezing temperature of spin-glass transition. Hence the material can be considered magnetically disordered at distances $r>k_{\mathrm{min}}^{-1} \equiv \xi_{\mathrm{mag}}$, where $\xi_{\mathrm{mag}}$ is the magnetic correlation length. This finite length is determined by the doping rather than by the temperature. Nevertheless, magnons with $k>k_{\min }$ still exist and the magnetic order at distances $r<\xi_{\text {mag }}$ is preserved (at least, at time scale $<\xi_{\text {mag }} / v_{s}$, where $v_{s}$ is the magnon velocity).

When the concentration grows up to $c>c_{0}$, where $c_{0}=\varepsilon_{0} / W$ is the characteristic concentration for insulator-metal transition ( $W$ is the whole conduction bandwidth), the Fermi level of free (hole) carriers is formed, manifesting the onset of metallic conductivity. It was shown that, within the scope of Lifshitz's impurity model, which is appropriate for HTSC materials, the $2 D$ systems are in fact more favorable (at small $c$ ) for this transition than the $3 D$ ones [17].

However, the two above mentioned problems were treated in Refs. 16-18 independently: the destroying of magnetic order was calculated with neglect of the band of free carriers (the indirect interaction between localized hole spins was only considered as mediated by the spin-wave band). On the other hand, the localized core spins were ignored as possible scatterers for electronic excitations in calculating the characteristic concentration $c_{0}$. Undoubtedly, a more conclusive description of HTSC compounds should take both factors into account simultaneously: scattering of free carriers on localized spins and indirect interaction between localized spins via the conduction band (in real cuprates, the valence band). This determines the purpose of the present work, to study the influence of magnetic order in cuprate layers on electronic processes at metallization.

The consideration below is restricted to only effects by magnon (Bloch-like) excitations with $k>k_{\min }$, leaving mostly aside the low-energy spin excitations which arenot described by the wavevector (spin fluctuation states). In other words, we consider the presence of magnon excitations in addition to the delocalized charge carriers, although the long-range magnetic order is absent. The main results of this analysis are the energy and temperature dependences for the magnon-controlled inverse lifetime $\Gamma$ of electronic quasiparticles near the Fermi level, specific for $2 D$ system. In particular, a linear temperature dependence $\Gamma(T)$ is found to be concentration independent and to begin from lower temperatures than follows from the known estimates in literature. This behavior can contribute considerably to the broadly discussed linear temperature resistivity in the normal phase of high- $T_{c}$ materials.

\section{Hamiltonian and Green functions}

We choose the basic model which joins in a simple way the models previously used for description of the magnetic [16] and electronic [17] parts of the HTSC system (bearing in mind for instance the compound $\left.\mathrm{La}_{2} \mathrm{CuO}_{4+\delta}\right)$ :

$$
\begin{gathered}
H=H_{\mathrm{el}}+H_{s}+H_{s-\mathrm{el}} \\
H_{\mathrm{el}}=\sum_{\mathbf{k}, \sigma} \varepsilon_{\mathbf{k}} a_{\mathbf{k}, \sigma}^{+} a_{\mathbf{k}, \sigma}-\frac{\Delta \varepsilon}{N} \sum_{\mathbf{p}, \mathbf{k}, \mathbf{k}^{\prime}, \sigma} \mathrm{e}^{i\left(\mathbf{k}-\mathbf{k}^{\prime}\right) \mathbf{p}} a_{\mathbf{k}, \sigma}^{+} a_{\mathbf{k}^{\prime}, \sigma}, \\
H_{s}=\sum_{\mathbf{q}, j} \Omega_{j}(\mathbf{q}) b_{\mathbf{q} j}^{+} b_{\mathbf{q} j} \\
H_{s-\mathrm{el}}=\sum_{\mathbf{k}, \mathbf{q}, j}\left[\gamma_{1}(\mathbf{q})\left(a_{\mathbf{k}, \uparrow}^{+} a_{\mathbf{k}-\mathbf{q}, \downarrow}-a_{\mathbf{k}, \downarrow}^{+} a_{\mathbf{k}-\mathbf{q}, \uparrow}\right)\left(b_{\mathbf{q} 1}-b_{-\mathbf{q} 1}^{+}\right)+\right. \\
\left.+\gamma_{2}(\mathbf{q})\left(a_{\mathbf{k}, \uparrow}^{+} a_{\mathbf{k}-\mathbf{q}, \downarrow}+a_{\mathbf{k}, \downarrow}^{+} a_{\mathbf{k}-\mathbf{q}, \uparrow}\right)\left(b_{\mathbf{q} 2}+b_{-\mathbf{q} 2}^{+}\right)\right] .
\end{gathered}
$$

Here $N$ is the number of elementary cells in the lattice, $a_{\mathbf{k}, \sigma}$ and $b_{\mathbf{q} j}$ are respectively the Fermi and Bose operators for electronic and magnon excitations. They are labelled by two-dimensional wavevectors $\mathbf{k}$ and $\mathbf{q}$, with $\sigma=\uparrow, \downarrow$ related to the carrier spin states and $j=1,2$ to FM-like and AFM-like magnon branches, and characterized by the isotropic dispersion laws in the long wavelength 
region: $\varepsilon_{\mathbf{k}}=\hbar^{2} \mathbf{k}^{2} / 2 m$ and $\Omega_{j}^{2}(\mathbf{q})=\Omega_{g j}^{2}+\hbar^{2} v_{s}^{2} \mathbf{q}^{2}$. The effective mass $m$, the magnon velocity $v_{s}$ and the spin-wave gaps $\Omega_{g j}$ can be expressed in terms of microscopic interaction parameters:

$$
\begin{gathered}
m=2 \hbar^{2} / t a^{2}, v_{s}=J a / \hbar, \\
\Omega_{g 1}=s z \sqrt{2 J \Delta J_{\mathrm{rh}}}, \Omega_{g 2}=s z \sqrt{2 J \Delta J_{t}} .
\end{gathered}
$$

For illustrative purposes, we shall use throughout this paper the parameter values for $\mathrm{La}_{2} \mathrm{CuO}_{4}$ : the oxygen-oxygen hopping matrix element $t \approx$ $\approx 0.6 \mathrm{eV}$, the AFM exchange constant $J \approx 0.1 \mathrm{eV}$, the rhombic anisotropy $\Delta J_{\mathrm{rh}} \approx 10^{-3} \mathrm{meV}$, the tetragonal anisotropy $\Delta J_{t} \approx 8 \cdot 10^{-3} \mathrm{meV}$, the $\mathrm{Cu}^{2+}$ spin $s=1 / 2$, the in-plane coordination number $z=4$, and magnetic lattice parameter $a \approx 5.4 \AA$. This gives $m$ of the order of the free electron mass, the bandwidth $W=\pi t \approx 2 \mathrm{eV}, \Omega_{g 1} \approx 1 \mathrm{meV}, \Omega_{g 2} \approx 2.5 \mathrm{meV}$, and $v_{s} \approx 10^{7} \mathrm{~cm} / \mathrm{s}$ (note that the latter value is comparable to typical values of the Fermi velocity $v_{F}$ in the metallic phase of HTSC compounds). Finally, $\Delta \varepsilon>0$ in (1) is the shift of the local oxygen level from its initial position because of Coulomb field of the doped ions (see Ref. 17).

The spin-electron coupling $H_{s-\text { el }}$ is derived from the standard Shubin-Vonsovsky model, using the specifics of spin structure for $\mathrm{La}_{2} \mathrm{CuO}_{4}[16]$, then the coupling parameters are

$$
\gamma_{j}(\mathbf{q}) \approx J^{\prime}\left(\frac{z \Omega_{j}(\mathbf{q})}{2 N J}\right)^{1 / 2},
$$

where $J^{\prime}$ is the exchange parameter between the core spin and a carrier spin at the nearest neighbor oxygen site (it is supposed to be of the order of or even greater than $J$ ). Thus, formally this interaction is quite similar to the common electron-phonon coupling in metals, which was studied in detail by Yu. Kagan and co-workers (see, for example, Ref. 21). However, as will be seen below, the model under consideration possesses certain specifics, due to the $2 D$ dispersion of either electron and magnon bands and to the absence of the usual adiabatic relation between the corresponding velocities. Of course, one can also derive the electronelectron coupling ( $d$-wave superconducting) from $H_{s-\mathrm{el}}$ but the analysis below will be restricted only to normal phases.

In addition to the above discussed translationally-invariant terms, the Hamiltonian, Eq. (1), namely, its $H_{\mathrm{el}}$ part, also contains the perturbation from dopants on oxygen sites $\mathbf{p}$, distributed chaotically with concentration $c$ (the minus sign before $\Delta \varepsilon$ accounts explicitly for attraction of carriers to charged dopants resulting in hole conductivity). In this study we focus mainly on the effects in electronic spectrum, so that impurity perturbations (such as, e.g., modification of the parameters $J$ and $J^{\prime}$ near impurity sites, due to the evident shifts of the on-site electronic levels) are not included in $H_{s}$ and $H_{s-\mathrm{el}}$. The corresponding effects can be accounted for implicitly by restricting the sums in the magnon wavevector to $q>k_{\min }$ and adding instead certain sums over (chaotic) spin fluctuation excitations. But to begin with such a so complicated problem, we shall at this first stage simply ignore the disorder effects in spin spectrum, and, only after obtaining the physical results, they will be checked with respect to the effects of spin disorder.

As usual, we shall infer the single-particle electronic properties from the Fourier-transformed twotime Green's functions (GF)

$$
g_{\mathbf{k}}(\varepsilon)=\left\langle\left\langle a_{\mathbf{k}, \sigma} \mid a_{\mathbf{k}, \sigma}^{+}\right\rangle\right\rangle_{\varepsilon}=i \int_{0}^{\infty} \mathrm{e}^{i(\varepsilon+i 0) t}\left\langle\left\{a_{\mathbf{k}, \sigma}(t), a_{\mathbf{k}, \sigma}^{+}\right\}\right\rangle d t,
$$

where $\{.,$.$\} is the anticommutator and \langle\ldots\rangle$ the quantum-statistical average. Their poles in the complex energy plane determine the energy spectrum and damping of quasiparticles.

A necessary pre-requisite for studying the impurity effects is a detailed knowledge of dynamics for the «background» uniform system (often this presents an independent problem in complex systems such as HTSC compounds). Hence, as a first step, we omit the impurity term even in $H_{\mathrm{el}}$. Then the equations of motion for GF, Eq. (4), to the lowest order in spin-electron coupling, readily yield the result:

$$
\begin{gathered}
g_{\mathbf{k}}(\varepsilon)=\left[\varepsilon-\varepsilon_{\mathbf{k}}-\Pi_{\mathbf{k}}(\varepsilon)\right]^{-1} \\
\Pi_{\mathbf{k}}(\varepsilon)= \\
=\sum_{\mathbf{q}, j} \gamma_{j}^{2}(\mathbf{q})\left(\frac{1-f_{|\mathbf{k}-\mathbf{q}|}+n_{\mathbf{q} j}}{\varepsilon_{\mathbf{k}}-\varepsilon_{|\mathbf{k}-\mathbf{q}|}-\Omega_{j}(\mathbf{q})}+\frac{f_{|\mathbf{k}-\mathbf{q}|}+n_{\mathbf{q} j}}{\varepsilon_{\mathbf{k}}-\varepsilon_{|\mathbf{k}-\mathbf{q}|}+\Omega_{j}(\mathbf{q})}\right)
\end{gathered}
$$
where $\quad f_{\mathbf{k}}=\left\{\exp \left[\left(\varepsilon_{\mathbf{k}}-\varepsilon_{F}\right) / T\right]+1\right\}^{-1} \quad$ and $n_{\mathbf{q} j}=\left\{\exp \left[\Omega_{j}(\mathbf{q}) / T\right]-1\right\}^{-1}$ are the Fermi and Bose occupation numbers, and $\varepsilon_{F}$ is the Fermi energy of the free holes; this coincides with the well-known expressions, e.g., from Ref. 22. Below we consider in detail how the properties of electron and magnon spectra, specific for our system, are reflected in the behavior of this otherwise comprehensively studied self-energy term $\Pi_{\mathbf{k}}(\varepsilon)$. Firstly we consider the 
broadening of electronic states close to the very Fermi level at zero temperature, due to inelastic electron-electron scattering with creation of magnons. Then we pass to the damping of electronic states on the Fermi level itself at finite temperatures, including processes with thermal magnons.

\section{Damping near the Fermi level at $T=0$}

As long as we neglect the impurity perturbation $\Delta \varepsilon$, but consider some finite doping level $c$, a well-defined Fermi level $\varepsilon_{F}=c W / 2$ exists in the $2 D$ electronic spectrum $[17,18]$. The broadening $\Gamma_{\mathbf{k}}$ of quasiparticle levels close to $\varepsilon_{F}$ is given by $\Gamma_{\mathbf{k}}=$ $=\operatorname{Im} \Pi_{\mathbf{k}}\left(\varepsilon_{\mathbf{k}}\right)$. However, bearing in mind that, in fact, the metallic state in HTSC compounds onsets well after the breakdown of long-range magnetic order, we should not consider quasiparticle energies closer to $\varepsilon_{F}$ than to the lower boundary energy of magnons, $\Omega_{\min }$. Since the latter value exceeds both spin-wave gaps $\Omega_{g j}$ [16], we can take the magnon dispersion law in the relevant region to be identical for the two branches and linear: $\Omega_{j}(\mathbf{q})=\Omega_{g} \approx \hbar v_{s} q$. Moreover, we extend this law to the whole magnon band, forgetting for a moment the long-wave limitation (we shall recall it at the end of this Section). Then, using Eq. (5) at $T=0$, and taking into account the $2 D$ character of spin-wave spectrum, we arrive at:

$$
\begin{gathered}
\Gamma_{\mathbf{k}}=\Gamma_{0} a \int_{0}^{q_{\max }} q d q \int_{0}^{2 \pi} d \varphi\left[\delta\left(2 k \cos \varphi-q+2 k_{s}\right) \times\right. \\
\times \theta\left(\varepsilon_{F}-\varepsilon_{\mathbf{k}}-\hbar v_{s} q\right)+ \\
\left.+\delta\left(2 k \cos \varphi-q-2 k_{s}\right) \theta\left(\varepsilon_{\mathbf{k}}-\varepsilon_{F}-\hbar v_{s} q\right)\right],
\end{gathered}
$$

where $\Gamma_{0}=4 \pi z J^{2} / W, k_{s}=\beta k_{F}$, and $\beta \equiv v_{s} / v_{F}$. The Fermi velocity depends directly on the doping level $c: v_{F}=v_{a} \sqrt{c}$, where $v_{a}=\sqrt{\pi / 2} a(t / \hbar)$, and, with our choice of parameters, $v_{a} \approx 7 \cdot 10^{7} \mathrm{~cm} / \mathrm{s}$. Then $v_{F}$ is close to $v_{s}$ (the ratio $\beta$ is close to unity) for $c$ being a few percent (this is in striking contrast with the usual situation for electron-phonon coupling in conventional metals where the sound velocity is about three orders of magnitude lower than $\left.v_{F}\right)$. After elementary integration, Eq. (6) yields an analytic result:

$$
\Gamma_{\mathbf{k}}=2 \Gamma_{0} a\left[\sqrt{k^{2}-k_{s}^{2}}-\sqrt{k^{2}-\left(k_{s}-q_{\mathbf{k}}\right)^{2}}+\right.
$$

$$
\begin{aligned}
& \left.+k_{s}\left(\arcsin \frac{k_{s}}{k}-\arcsin \frac{k_{s}-q_{\mathbf{k}}}{k}\right)\right] \times \\
& \quad \times \theta\left(k-k_{s}\right) \theta\left(k-k_{s}+q_{\mathbf{k}}\right),
\end{aligned}
$$

where $q_{\mathbf{k}}=\left(\varepsilon_{\mathbf{k}}-\varepsilon_{F}\right) /\left(2 \beta \hbar v_{F}\right)$. The $\theta$-functions in Eq. (7) determine that $\Gamma_{\mathbf{k}}$ drops to zero below certain threshold quasimomentum value, namely: for $k<\left(8 k_{s}^{2}+k_{F}^{2}\right)^{1 / 2}-2 k_{s}$ if $\beta<1$, and for $k<k_{s}$ if $\beta>1$. Close to the Fermi surface $($ at $\beta<1)$ we obtain a quadratic dependence of $\Gamma_{\mathbf{k}}=\Gamma(\varepsilon)$ on the quasiparticle energy $\varepsilon=\varepsilon_{\mathbf{k}}-\varepsilon_{F}$ :

$$
\Gamma(\varepsilon) \approx \frac{\Gamma_{0}}{a k_{F} \sqrt{1-\beta^{2}}}\left(\frac{\varepsilon}{4 J}\right)^{2},
$$

which is characteristic of $2 D$ dispersion, unlike the usual $\varepsilon^{3}$ law in $3 D$ systems [22]. Note that, since the relation $a k_{F}=\sqrt{2 \pi c}$, this function increases with decreasing concentration. In no way does the presence of the enhancement factor $\left(1-\beta^{2}\right)^{-1 / 2}$ in the latter formula mean divergence of $\Gamma(\varepsilon)$ in the «relativistic» limit $\beta \rightarrow 1$ (reached when the concentration decreases down to $\left.c \rightarrow c_{s}=2 \pi(J / W)^{2}\right)$ : in this case the quadratic law, Eq. (8), is simply changed for a $\varepsilon^{3 / 2}$ law: $\Gamma\left(\varepsilon, c_{s}\right) \approx 0.6\left(J^{\prime} / J\right)^{2} W^{-1 / 2} \varepsilon^{3 / 2}$. With the parameter values adopted in Sec. 2 , we come to the conclusion (at least, when $T=0$ ) that the usual Fermi-liquid condition $\Gamma(\varepsilon) / \varepsilon<1$ is well assured for the considered magnon-induced damping in a rather broad vicinity of the Fermi surface at all relevant concentrations $c>c_{0}$.

To conclude this Section, we estimate the region of validity of Eqs. (7), (8) with respect to the above mentioned neglection of spin fluctuation states. In fact, the integration over $q$ in Eq. (6) is only legal for $q>k_{\min }$ (see Sec. 1), hence the result, Eq.

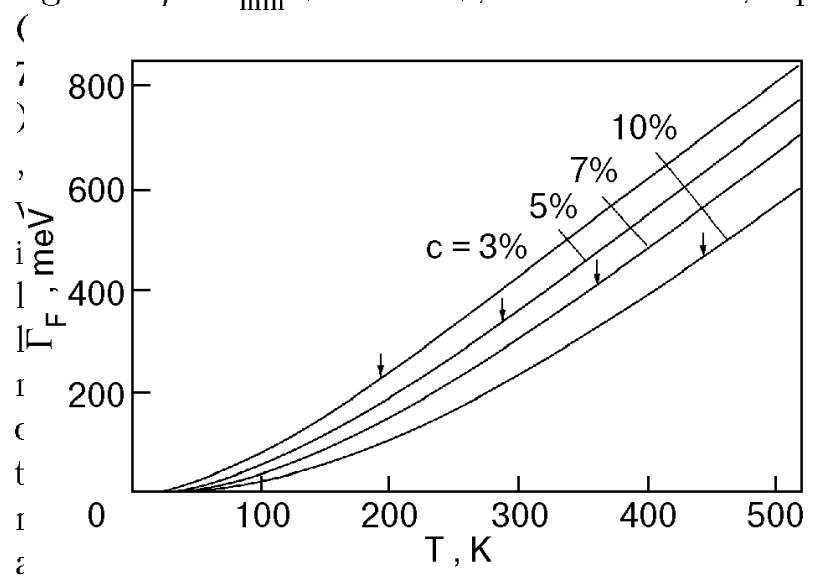

Kig. 1. Temperature dependence of quasiparticle broadening at the Fermi level for different dopant concentrations. The arrows Sindicate the calculated values of crossover temperature $T_{c-o}$, dbut the visible linearity extends to even lower temperatures.

n 
se if $q_{\mathbf{k}} \lesssim 2 k_{\text {min }}$. Then, with the estimate $k_{\min } \sim c\left(J^{\prime} / J\right)^{2} a^{-1}$ for a minimum magnon wavevector at $T=0$ [16] the formula, Eq. (8), is found to fail only within a narrow fluctuation region: $|\varepsilon| \lesssim \varepsilon_{f} \sim c J^{2} / J$. At the boundary of this region, we have the Fermi-liquid ratio $\Gamma\left(\varepsilon_{f}\right) / \varepsilon_{f} \sim$ $\sim \sqrt{\pi c / 2} J^{\prime 4} / W J^{3}$, which is considerably smaller than unity. At least, the simplest assertion on the behavior of $\Gamma$ within the fluctuation region is obtained by changing the «magnon density of states» factor $q$ in Eq. (6) by a constant value $\sim k_{\text {min }}$ at $q<k_{\min }$, which gives $\Gamma(\varepsilon) \sim \Gamma\left(\varepsilon_{f}\right)$ at $|\varepsilon| \lesssim \varepsilon_{f}$, when it is impossible to speak about undamped quasiparticles.

\section{Damping of Fermi states at finite temperatures}

Using explicitly the $\delta$-function relations in the occupation numbers $f_{|\mathbf{k}-\mathbf{q}|}$ and $n_{\mathbf{q} j}$ in $\operatorname{Im} \Pi_{\mathbf{k}}\left(\varepsilon_{\mathbf{k}}\right)$ at $\varepsilon_{\mathbf{k}}=\varepsilon_{F}$, the temperature dependent broadening at the very Fermi level can be obtained from Eq. (5) in the simple form:

$$
\Gamma_{F}(T)=2 \Gamma_{0} a k_{F}\left[\int_{0}^{1-\beta} \frac{x d x}{\sinh \left(2 J a k_{F} x / T\right) \sqrt{1-(\beta+x)^{2}}}+\int_{0}^{1+\beta} \frac{x d x}{\sinh \left(2 J a k_{F} x / T\right) \sqrt{1-(\beta-x)^{2}}}\right] .
$$

This expression exhibits a crossover from quadratic function of temperature:

$$
\Gamma_{F}(T) \approx \frac{\Gamma_{0}}{2 a k_{F} \sqrt{1-\beta^{2}}}\left(\frac{\pi T}{J}\right)^{2}
$$

at low temperatures: $T<<2 J a k_{F}$ (which decreases as a function of $c$ alike $\Gamma(\varepsilon)$, Eq. (8)), to linear function of temperature at $T>>2 J a k_{F} \equiv 2 \Omega_{j}\left(\mathbf{k}_{F}\right)$ which, remarkably, is concentration independent:

$$
\Gamma_{F}(T) \approx \Gamma_{0} \frac{\pi T}{J} .
$$

Such a crossover is rather clear, if we notice that the thermal magnon wavevector $q_{T}=T / \hbar v_{S}$ exceeds the diameter $2 k_{F}$ of the Fermi circle just at $T>2 J a k_{F}$, but the independence of Eq. (11) on $\mathbf{k}_{F}$ is another characteristic feature of $2 D$ dispersion, also in striking contrast with $3 D$ systems. The crossover temperature found from comparison of the values, Eqs. (10), (11), is $T_{c-o}=2 J a k_{F} \sqrt{1-\beta^{2}} / \pi$. In fact, this crossover, as seen from direct numeric calculations, using Eq. (9) and the parameter values chosen in Sec. 2 (Fig. 1), occurs even earlier, at about $0.6 T_{c-o}$, which is essentially lower than the commonly considered value $T_{c-o} \sim J$ [23].

Taking into mind the estimate, in the end of the previous section, for the fluctuation limit $\Gamma\left(\varepsilon_{f}\right)$ of the broadening close to the Fermi level, it follows that this constant limit can be reached for $\Gamma_{F}(T)$, with lowering temperature, at some value $T_{f} \sim \sqrt{c}\left(J^{\prime} / J\right) T_{c \rightarrow 0}$, which is yet much lower than the crossover temperature value.

\section{Acknowledgments}

We are pleased to devote this paper to the seventieth anniversary of Prof. Yu. Kagan whose contribution to the physics of disordered systems in general and high- $T_{c}$ superconductors in particular cannot be overstimated. Taking this opportunity we would like to acknowledge Prof. Yu. Kagan for his numberless discussions and benevolent criticisms of our results which always were useful and helpful for us.

One of us (Yu. G. P.) thanks the support from the Portuguese Program PRAXIS XXI through the project $\mathrm{BCC} / 6428 / 95$.

1. R. J. Birgeneau and G. Shirane, in: Physical Properties of High-T Superconductors, I, 151 (1990).

2. D. Vakhnin, S. K. Sinha, D. E. Monston, D. C. Johnston, J. M. Newsom, C. R. Safenya, and J. H. E. King, Phys. Rev. Lett. 58, 2802 (1987).

3. T. Thio, T. R. Thurston, N. W. Preyer, P. J. Picone, M. A. Kastner, H. P. Jenssen, D. R. Gabbe, C. Y. Chen, R. J. Birgeneau, and A. Aharony, Phys. Rev. B38, 905 (1988).

4. S.-W. Cheong, Z. Fisk, and J. O. Willis, Solid State Commun. 65, 111 ( 1988).

5. H. Fai Fong, Phys. Rev. Lett. 75, 316 (1995).

6. S. M. Hayden, G. Aeppli, H. A. Mook, T. G. Perring, I. E. Mason, S.-W. Cheong, and Z. Fisk, Phys. Rev. Lett. 76, 1344 (1996).

7. G. Shirane, R. J. Birgeneau, Y. Endoh, and M. A. Kastner, Physica B197, 158 (1994).

8. V. M. Loktev, Fiz. Nizk. Temp. 22, 3 (1996) [Low Temp. Phys. 22, 1 (1996)].

9. N. W. Preyer, R. J. Birgeneau, C. Y. Chen, D. R. Gabbe, H. P. Jenssen, M. A. Kastner, P. J. Picone, and T. Thio, Phys. Rev. B39, 11563 (1989).

10. J. P. Flack, A. Levy, M. A. Kastner, and R. J. Birgeneau, Phys. Rev. Lett. 69, 1109 (1992).

11. Yu. A. Izyumov, Sov. Phys. Usp. 165, 403 (1995).

12. W. E. Pickett, Rev. Mod. Phys. 61, 433 (1989). 
13. A. P. Kampf, Phys. Rep. 249, 219 (1994).

14. W. Brenig, Phys. Rep. 251, 153 (1995).

15. D. Pines, Tr. J. Phys. 20, 535 (1996).

16. M. A. Ivanov, V. M. Loktev, and Yu. G. Pogorelov, Sov. Phys. JETP 74, 317 (1992).

17. M. A. Ivanov, V. M. Loktev, Yu. G. Pogorelov, and Yu. V. Skripnik, Fiz. Nizk. Temp. 17, 716 (1991) [Sov. J. Low Temp. Phys. 17, 377 (1991)].

18. M. A. Ivanov, V. M. Loktev, and Yu. V. Skripnik, Fiz. Nizk. Temp. 22, 1186 (1996) [Low Temp. Phys. 22, 907 (1996)].
19. V. M. Loktev and Yu. G. Pogorelov, Fiz. Nizk. Temp. 22, 1018 (1996) [Low Temp. Phys. 22, 776 (1996)].

20. V. M. Loktev, Sverkhprovod.: Fiz., Khim. Tekhn. 4, 2293 (1991).

21. Yu. M. Kagan and A. P. Zhernov, Sov. Phys. JETP 23, 737 (1966).

22. L. D. Landau and E. M. Lifshitz, Course of Theoretical Physics, Vol. 9: E. M. Lifshitz and L.P. Pitaevskii, Statistical Physics, Part II, Pergamon Press, Oxford (1980).

23. H. Kohno and K. Yamada, Progr. Theor. Phys. 85, 13 (1991). 